\title{
Subthreshold Dynamics in Periodically Stimulated Squid Giant Axons
}

\author{
Daniel T. Kaplan, ${ }^{1,2}$ John R. Clay, ${ }^{3,4}$ Timothy Manning, ${ }^{1}$ Leon Glass, ${ }^{1,2}$ Michael R. Guevara, ${ }^{1,2}$ and Alvin Shrier ${ }^{2,4}$ \\ ${ }^{1}$ Centre for Nonlinear Dynamics, McGill University, Montreal, Quebec, Canada H3G 1 Y6 \\ ${ }^{2}$ Physiology Department, McGill University, Montreal, Quebec, Canada H3G 1 Y6 \\ ${ }^{3}$ Laboratory of Neurophysiology, National Institutes of Health, Bethesda, Maryland 20892 \\ ${ }^{4}$ Marine Biological Laboratory, Woods Hole, Massachusetts 02543
}

(Received 27 December 1995)

\begin{abstract}
Action potentials resulting from periodic stimulation of nerve axons occur at intervals that are irregular at moderate stimulation frequencies. Histograms of the intervals are multimodal, as seen in stochastic resonance. At higher stimulation frequencies, the action potentials are suppressed entirely, leaving only subthreshold dynamics. Return maps constructed from data show that both types of response are governed by the same deterministic one-dimensional description, with an unstable subthreshold fixed point largely accounting for the irregular intervals at moderate stimulation frequencies. [S0031-9007(96)00200-1]
\end{abstract}

PACS numbers: 87.22.Jb, 05.40.+j, 05.45.+b, 87.10.+e

Excitable systems play a fundamental role in neural information processing and many other biological systems. Often, one measures only large amplitude suprathreshold activity, for instance, the action potential (AP) in nerve or ventricular contraction in heart. An interesting feature of such activity is that in response to a periodic input, the times between these large suprathreshold events can, when plotted in histograms, produce peaks separated by multiples of the input's period [1,2]. A recent hypothesis is that such distributions may be associated with stochastic resonance [3]. In this paper, we show that the suprathreshold dynamics of two excitable systems - one living and one a simple mathematical model-display similar behavior. In these systems, however, the irregular suprathreshold response is associated with deterministic subthreshold chaos. This chaos can be identified as such only if the subthreshold events that occur between suprathreshold activity are also analyzed.

Experiments were performed on giant axons $(\approx 500 \mu \mathrm{m}$ diameter) of the common North Atlantic squid (Loligo pealei) using standard techniques [4]. Figure 1 shows the effect of injecting a periodic train of current pulses into an axon. The interpulse interval $T$ (in $\mathrm{ms}$ ) is indicated at the right of each panel, and is systematically decreased in successive runs. For $T$ sufficiently large, each stimulus pulse elicits an action potential, resulting in a stimuli:AP ratio of 1:1 [Fig. 1(a)]. As $T$ is decreased, one eventually loses 1:1 synchronization and encounters rhythms containing both APs and subthreshold responses [5-7]. For example, a 2:1 rhythm is observed at $T=26 \mathrm{~ms}$ [Fig. 1(b)], and a $3: 1$ rhythm at $T=17 \mathrm{~ms}$ [Fig. 1(c)]. For $T \leq 16 \mathrm{~ms}$ [Figs. 1(d)-1(h)], more complex rhythms, which can be aperiodic and may contain graded action potentials [e.g., second AP in Fig. 1(d)], are seen. As $T$ is decreased, the frequency of occurrence of APs relative to subthreshold responses tends to decline, until, for $T<12.0 \mathrm{~ms}$, all responses are subthreshold, with APs no longer being generated [Figs. 1(i)-1(1)] except for a single AP at the first stimulus of the train. For $T=11.8 \mathrm{~ms}$ [Fig. 1(i)], the subthreshold responses are themselves aperiodic. For $T=$ $11.6 \mathrm{~ms}$ [Fig. 1(j)] and $T=11.4 \mathrm{~ms}$ [Fig. 1(k)], there is a periodic subthreshold 2:0 rhythm consisting of a longerlasting subthreshold response alternating with a briefer subthreshold response. At the fastest stimulation rates ( $T \leq 11.2 \mathrm{~ms}$ ) there is 1:0 rhythm [Fig. 1(l)].

Given the variety of different shapes of APs and subthreshold responses in Fig. 1, we characterize the response to each stimulus by the area under the resulting deflection

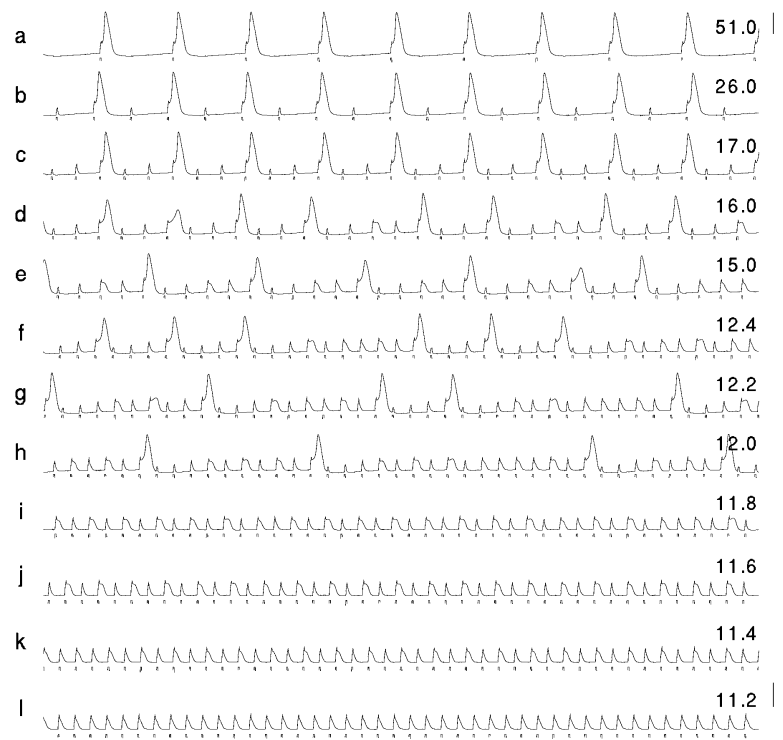

FIG. 1. Transmembrane potential of a squid giant axon in response to delivery of a periodic train of current pulses. Interval in ms between pulses $T$ is given to the right of each $0.5 \mathrm{~s}$ record. The train of current pulse stimuli is shown underneath each voltage trace. Traces illustrated were made after transients passed. The axon was allowed to rest for at least $3 \mathrm{~s}$ between runs. Pulse amplitude $=0.6 \mathrm{~mA}$, pulse duration $=1 \mathrm{~ms}$. Vertical calibration bar in (a) indicates -50 to $0 \mathrm{mV}$. Similar results were seen in ten other axons. 

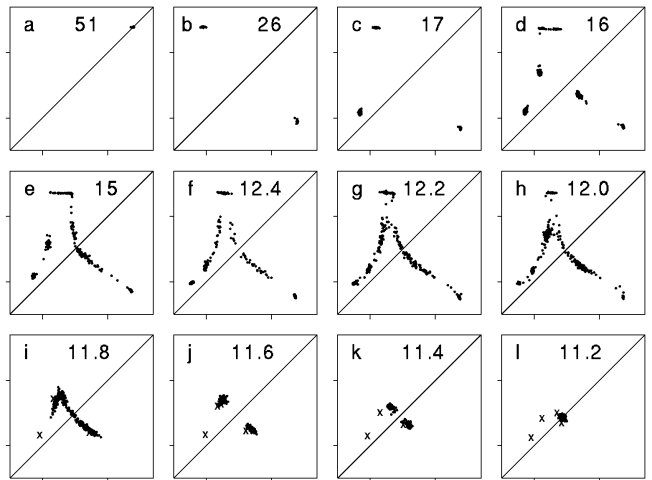

FIG. 2. Return maps constructed from experimental runs shown in Fig. 1. The interval $T$ between pulses (in $\mathrm{ms}$ ) is given in each panel. A baseline to the voltage wave forms of Fig. 1 was constructed by linear interpolation between segments of the voltage tracing bracketing a response. The area $(A)$ under each deflection (i.e., between the voltage tracing and the interpolated baseline) was calculated. 300 successive pairs of computed points $\left(A_{i+1}, A_{i}\right)$ were plotted on a logarithmic (base 10) scale. The first few points, indicated by $\times$, show the startup transients. The area is given in arbitrary units.

in the voltage trace. We then plot the area under the $i+1$ response $\left(A_{i+1}\right)$ vs the area under the preceding response $\left(A_{i}\right)$. Figure 2 shows the "return maps" thus created for each of the runs of Fig. 1. A logarithmic scale is used because of the large difference between the area of an AP and a subthreshold response. Use of the area rather than, for example, maximum voltage helps to reduce noise and, importantly, represents the overall shape of the subthreshold responses, allowing responses showing similar peak excursions in voltage to be distinguished. The maps shown in Fig. 2 do not depend strongly on the precise nature in which the areas are calculated.

The return maps in Fig. 2 suggest that all the irregular rhythms are governed by a family of underlying deterministic one-dimensional maps that have the shape of a " $\Lambda$ " or "volcano." The underlying map changes only slightly in form from one panel to the next, although the observed dynamics change qualitatively, from a stable subthreshold fixed point [Fig. 2(1)] to a stable period-2 orbit [Figs. 2(j) and 2(k)] to a subthreshold irregular, potentially chaotic, pattern [Fig. 2(i)]. Irregular patterns are also seen in Figs. 2(e)-2(h), all of which involve occasional action potentials. Figures 2(b) and 2(c) show stable periodic orbits involving APs, while Fig. 2(a) shows a stable suprathreshold fixed point where every stimulus elicits an AP. Although the stable periodic orbits of Figs. 2(j)-2(l) mark only one or two points on the volcano-shaped map, the start-up transients (marked as $\times$ ) clearly sketch out the other regions of the map.

Figure 2 shows a continuous progression in the shape of the volcano map as the pacing interval $T$ is changed. The dynamics in the presence of action potentials [Figs. 2(b)2(h)] are remarkably similar to dynamics in the absence of APs. (If there is a stable periodic orbit of low period [e.g.,
Figs. 2(a)-2(c)], the whole volcano cannot be visualized.) There is a fixed point where the right flank of the volcano intersects the line $A_{i+1}=A_{i}$. In Figs. 2(f)-2(i), the slope of the map at this fixed point is only slightly steeper than -1 ; hence the dynamics diverge slowly from the unstable fixed point. This results in the long intervals between APs seen in Figs. 1(f)-1(h). Since the same unstable fixed point appears in the presence and absence of action potentials, we conclude that the fixed point does not reflect steady, ultraslow recovery from an AP, but rather the ability of successive stimuli to prolong recovery from a previous AP. When the fixed point is stable, this prolongation can be renewed indefinitely.

Stochastic membrane noise generated by the opening and closing of single channels in the membrane [3] undoubtedly contributes to some of the irregularity in the rhythms of Fig. 1. For example, the data points in the return maps corresponding to periodic rhythms [Figs. 2(a)-2(c) and 2(j)-2(1)] form small clusters rather than superimposing exactly upon one another.

In order to investigate the possible contribution of chaotic dynamics to the irregular rhythms described above, we examined the behavior of a simple deterministic model of the squid axon, the FitzHugh-Nagumo (FHN) model [8]

$$
\begin{aligned}
& \frac{d v}{d t}=-v(v-1)(v-a)-w+I, \\
& \frac{d w}{d t}=c(v-b w),
\end{aligned}
$$

where $v$ has characteristics of membrane potential and excitability, $w$ is related to recovery or refractoriness, $I$ represents the injected stimulus current, and $a=0.139$, $b=2.54, c=0.008$ [9]. The FHN model has been shown to display stochastic resonance in the presence of noise [2,3], but here we study it with no noise. The response of the FHN model to periodic stimulation is shown in Fig. 3, with time series shown in the upper panels and return maps in the lower panels. As $T$ is decreased, one obtains periodic rhythms containing APs [Fig. 3(a)], irregular rhythms containing APs [Fig. 3(b)], irregular subthreshold rhythms [Figs. 3(c) and 3(d)], a 4:0 rhythm [Fig. 3(e)], a 2:0 rhythm (not shown), and eventually a 1:0 rhythm [Fig. 3(f)] with only the first stimulus in a periodic train eliciting an AP. Once again, the lower panels indicate that there is a deterministic one-dimensional volcanoshaped map underlying the dynamics. When analyzing the FHN model, we have additional freedom to construct maps based on the recovery variable $w$. These maps (not shown) display an unstable fixed point corresponding to that seen in the maps $A_{i+1}$ vs $A_{i}$. Since recovery variables are not experimentally accessible in the squid preparation, we have used $A_{i}$ to characterize both model and experiment.

In prior experimental $[10,11]$ and modeling [12-14] work, it has been possible to delineate the full form of a one-dimensional map using a nonperiodic stimulation 

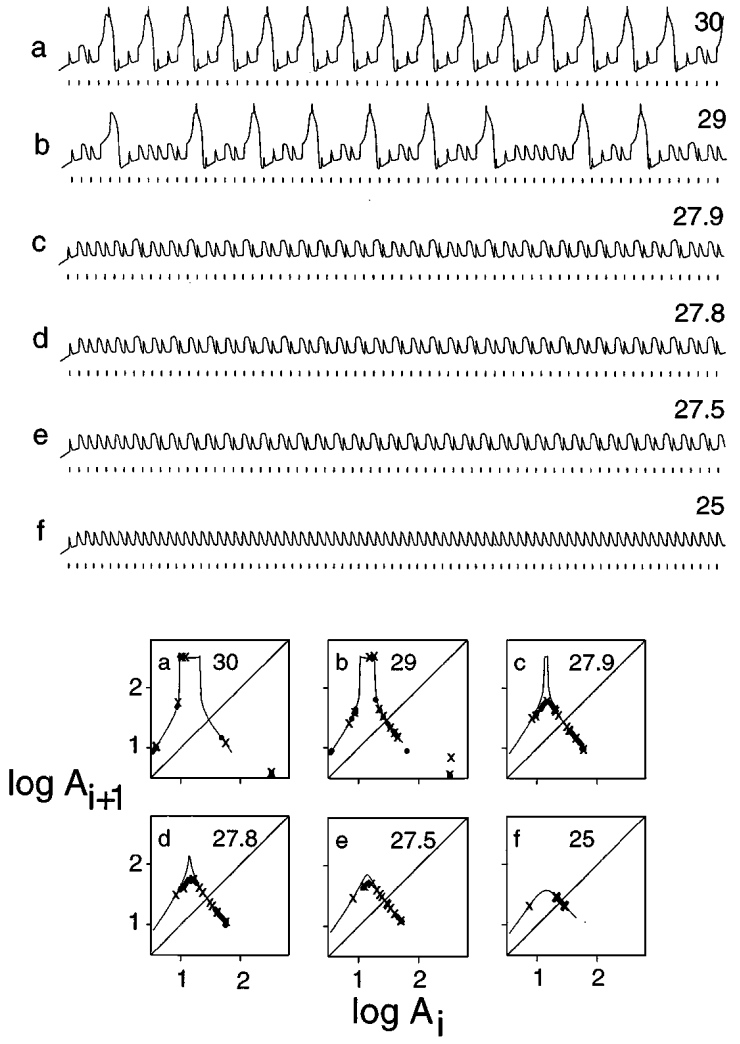

FIG. 3. Upper panels: Traces of the $v$ variable in the FitzHugh-Nagumo model. Interstimulus interval $(T)$ given to the right of each panel. Stimulus pulse train shown below each trace. Lower panels: Return maps from the FitzHughNagumo model constructed from the traces shown in upper panels (2000 time units). Interstimulus interval $T$ given in the upper right-hand corner of each panel. Data points $\left(A_{i+1}, A_{i}\right)$ from periodic stimulation of the model. Transients marked as $\times$ for first 11 points and $\bullet$ thereafter. The continuous curves show results from the three-pulse protocol applied to the model as described in the text. The area is given in arbitrary units.

protocol. To draw the full form of the map for the FHN model, we carried out simulations using a three-pulse protocol. The first stimulus pulse elicits an AP. The second and third stimuli are delivered at variable times following the first stimulus. The curves in the return maps of Fig. 3 plot the area of the response induced by the third stimulus pulse as a function of the area of the response induced by the second pulse. It is clear that the response to periodic stimulation $(\times, \bullet)$ lies close to this curve, indicating that this response is, in fact, controlled by an approximately one-dimensional process. The discrepancy between the points and the curves, especially in panels (c)(e), stems from the fact that the model has two variables, whereas only a single parameter (the area) is used here to describe the response to the three-pulse protocol.

Interspike interval (ISI) histograms from both the squid axon and the FHN model are multimodal (Fig. 4). Multimodality in related settings has been the topic of much recent discussion $[1-3,15,16]$. While the finite
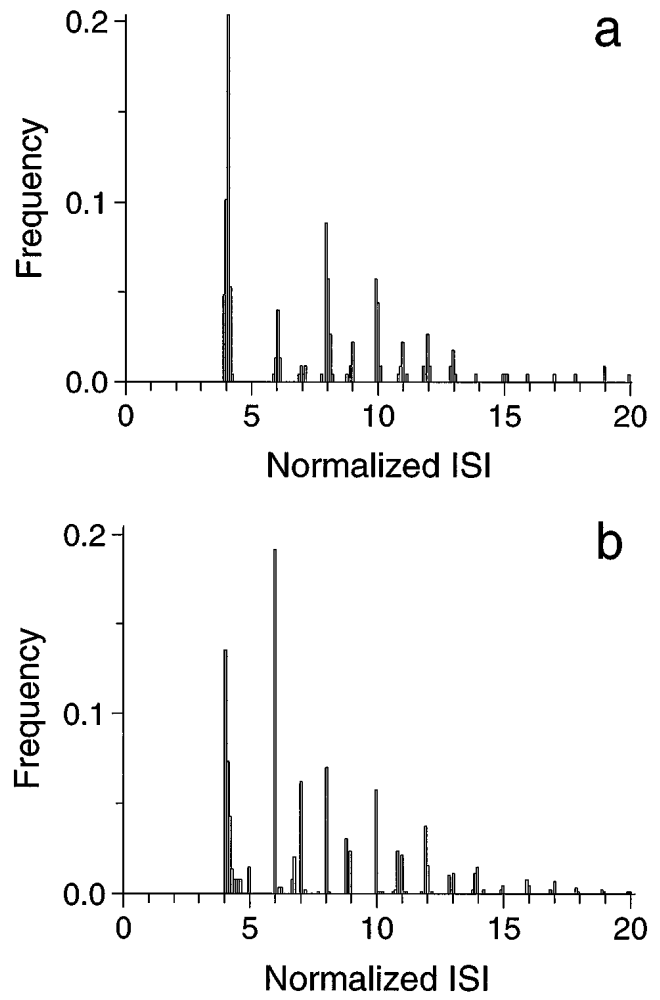

FIG. 4. Interspike interval (ISI) histograms from squid axon and FitzHugh-Nagumo model. (a) Squid axon: data taken from the run, part of which is shown as Fig. $1(\mathrm{~g})(T=12.2 \mathrm{~ms})$. (b) FitzHugh-Nagumo model: data taken from simulations of Fig. 3 ( $T=29.5$ time units). In both (a) and (b), intervals are calculated between peaks of action potentials and are normalized to the interstimulus interval.

width of peaks in such histograms is usually attributed to stochastic membrane noise (see, e.g., [17]), our work points out a new deterministic mechanism by which APs are generated at times that are roughly, but not exactly, multiples of a basic interspike interval. The finite width of the peaks is due to the graded nature of the AP; the details of the structure within a peak are not understood but depend to some extent on the criterion used to define the occurrence on an AP.

It is well known that subthreshold responses can play an important role in "conditioning" the response of neurons to subsequent stimuli [18-21]. In fact, these subthreshold responses are not merely passive events, but active ones that have been termed "local responses" [21]. Our work shows explicitly that subthreshold responses play a crucial role in generating complex aperiodic sequences of intermixed subthreshold responses and action potentials. While there have been several reports of period-doubling bifurcations and chaotic dynamics in squid axon [6,7] and the FHN equations [12-14,22,23], we are not aware of any prior descriptions of subthreshold period doubling and chaos in response to injection of a periodic pulse train in these systems. By constructing return maps that plot the area of both subthreshold and suprathreshold responses, one can 
characterize the state of the system in a way that unifies the subthreshold and suprathreshold phenomenology. This shows that both subthreshold and suprathreshold dynamics display a fixed point: a novel deterministic mechanism for the generation of irregular spike trains.

This work was supported by an operating grant from the Medical Research Council (Canada) and the Fonds de la recherche en santé du Québec. We thank Adam Sherman for software development and David Miller for help with data analysis.

[1] A. Longtin, A. Bulsara, and F. Moss, Phys. Rev. Lett. 67, 656-659 (1991).

[2] A. Longtin, Chaos 5, 209-215 (1995).

[3] K. Wiesenfeld and F. Moss, Nature (London) 373, 33-36 (1995).

[4] J. R. Clay and M. F. Shlesinger, Biophys. J. 42, 43-53 (1983). The intracellular perfusate consisted of $250 \mathrm{mM}$ K-glutamate, $50 \mathrm{~m} M \mathrm{KF}, 40 \mathrm{~m} M$ Na-glutamate, and $400 \mathrm{~m} M$ sucrose $(p \mathrm{H}=7.3)$. The extracellular solution was filtered seawater. The temperature was $8-12{ }^{\circ} \mathrm{C}$, kept constant to within $0.1{ }^{\circ} \mathrm{C}$ within any single experiment. The transmembrane potential was measured with glass microelectrodes filled with $0.5 \mathrm{M} \mathrm{KCl}$ and $1 \%$ agar placed inside and outside of the axon. Current pulses were injected into the axon using an axially placed platinum wire in series with a $10 \mathrm{M} \Omega$ resistor, which determined the amplitude of the current pulse [since the input resistance of the axon was $\approx 2 \mathrm{k} \Omega(\ll 10 \mathrm{M} \Omega)]$. A software package (Alembic Software Co., Montreal, Canada) run on an 80386 PC implemented the stimulation protocols. Under these conditions, the resting potential and APs were stable for several hours.

[5] R. Guttman, L. Feldman, and E. Jakobsson, J. Membr.
Biol. 56, 9-18 (1980).

[6] G. Matsumoto, N. Takahashi, and Y. Hanyu, in Chaos in Biological Systems, edited by H. Degn, A. V. Holden, and L. F. Olsen (Plenum, New York, 1987), pp. 143-156.

[7] N. Takahashi, Y. Hanyu, T. Musha, R. Kubo, and G. Matsumoto, Physica (Amsterdam) 43D, 318-334 (1990).

[8] R. FitzHugh, Biophys. J. 1, 445-466 (1961).

[9] J. Rinzel, in Research Notes in Mathematics-Nonlinear Diffusion, edited by W.E. Fitzgibbon and H. R. Walker (Pittman Press, London, 1977), pp. 186-212.

[10] M. R. Guevara, L. Glass, and A. Shrier, Science 214, 1350-1353 (1981).

[11] D. Chialvo, R.F. Gilmour, Jr., and J. Jalife, Nature (London) 343, 653-657 (1990).

[12] M. R. Guevara, L. Glass, M.C. Mackey, and A. Shrier, IEEE Trans. Syst. Man. Cybern. SMC-13, 790-798 (1983).

[13] A. Rabinovitch, R. Thieberger, and M. Friedman, Phys. Rev. E 50, 1572-1578 (1994).

[14] S. J. Doi and S. Sato, Math. Biosci. 125, 229-250 (1995).

[15] J. K. Douglass, L. Wilkens, E. Pantazelou, and F. Moss, Nature (London) 365, 337-340 (1993).

[16] H. A. Braun, H. Wissing, K. Schaefer, and M. C. Hirsch, Nature (London) 367, 270-273 (1994).

[17] K. Wiesenfeld, D. Pierson, E. Pantazelou, C. Dames, and F. Moss, Phys. Rev. Lett. 72, 2125-2129 (1994).

[18] E. D. Adrian and K. Lucas, J. Physiol. (London) 44, 68124 (1912).

[19] A. V. Hill, Proc. R. Soc. London B 119, 305-355 (1936).

[20] B. Katz, Proc. R. Soc. London B 124, 244-276 (1938).

[21] B. I. Khodorov, The Problem of Excitability (Plenum Press, New York and London, 1974), pp. 37-52.

[22] M. Feingold, D. L. Gonzalez, O. Piro, and H. Viturro, Phys. Rev. A 37, 4060-4063 (1988).

[23] S. Rajasekhar and M. Lakshmanan, J. Theor. Biol. 133, 473-477 (1988). 